\title{
Cold-adapted protease enables quantitation of surface proteins in the absence of membrane trafficking
}

\section{Protocol For: \\ Cold-adapted protease enables quantitation of surface proteins in the absence of membrane trafficking}

Faraz Ahmad', Sarah Kate Coleman², Kai Kaila1,3, and Peter Blaesse ${ }^{1}$

${ }^{1}$ Laboratory of Neurobiology, University of Helsinki, Helsinki, Finland, 2Division of Biochemistry

and Biotechnology, University of Helsinki, Helsinki, Finland, and ${ }^{3}$ Neuroscience Center,

University of Helsinki, Helsinki, Finland

BioTechniques Protocol Guide, January 2017; doi 10.2144/000114502

See full protocol online: https://benchtalk.biotechniques.com/users/8521-biotechniques-editors/posts/11620-protocol-for-cold-adapted-proteaseenables-quantitation-of-surface-proteins-in-the-absence-of-membrane-trafficking

Protocol Summary: We report here an improved method for analyzing protein surface expression utilizing a cold-adapted trypsin. Preservation of activity of the enzyme at $0-4^{\circ} \mathrm{C}$ permits modification of the protease method of surface analysis to temperatures at which trafficking of mammalian plasmalemmal proteins is blocked. This is an important advantage over established trypsin-cleavage protocols. Moreover, the method is less time-consuming than surface biotinylation.

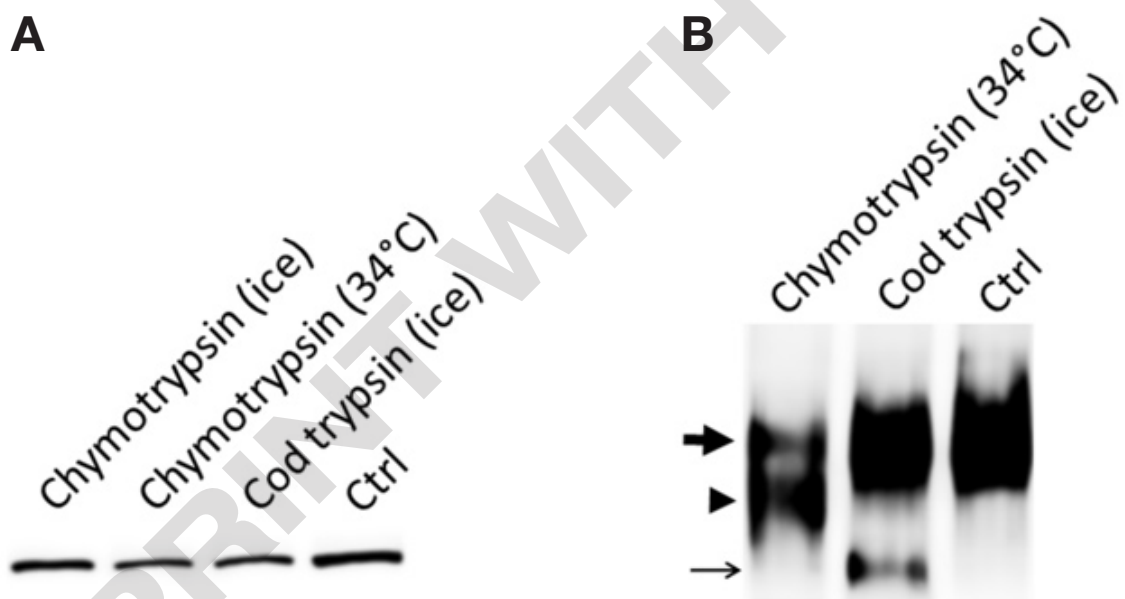

Protocol Schematic: Comparison of cod trypsin and mammalian chymotrypsin proteolysis of surface proteins. (A) Hippocampal slices were treated with identical concentrations of cod trypsin on ice and with bovine chymotrypsin at $34^{\circ} \mathrm{C}$ and on ice for $60 \mathrm{~min}$ and compared with the untreated control slices. Homogenization of the slices, electrophoresis, immunoblotting, and densitometry were carried out as described elsewhere (5). While there was only a slight proteolysis of GluA4 by bovine chymotrypsin on ice, the proteolytic activity of cod trypsin on ice and chymotrypsin at $34^{\circ} \mathrm{C}$ are comparable. (B) Hippocampal slices were treated with identical concentrations of cod trypsin on ice and with bovine chymotrypsin at $34^{\circ} \mathrm{C}$ for 60 min. Untreated slices served as a control. The chymotrypsin and trypsin proteolytic cleavage products of KCC2 were identified at different sizes and are denoted by an arrowhead and an arrow, respectively. There was a large decrease in the full-length KCC2 signal (denoted by block arrow) for slices treated with bovine chymotrypsin at $34^{\circ} \mathrm{C}$ compared with the slices treated with cod trypsin on ice. Correspondingly, the immunoreactive signal for the cleavage product was much more intense after treatment with bovine chymotrypsin at $34^{\circ} \mathrm{C}$. 